ARTICLE

Received 18 Oct 2015 | Accepted 12 Jul 2016 | Published 23 Aug $2016 \quad$ DOl: 10.1038/ncomms12552 OPEN

\title{
Hybrid bio-photo-electro-chemical cells for solar water splitting
}

\author{
Roy I. Pinhassi ${ }^{1, \star}$, Dan Kallmann ${ }^{1, \star}$, Gadiel Saper ${ }^{1, \star}$, Hen Dotan $^{2}$, Artyom Linkov ${ }^{3}$, Asaf Kay ${ }^{2}$, Varda Liveanu ${ }^{4}$, \\ Gadi Schuster ${ }^{4}$, Noam Adir $^{3} \&$ Avner Rothschild ${ }^{2}$
}

Photoelectrochemical water splitting uses solar power to decompose water to hydrogen and oxygen. Here we show how the photocatalytic activity of thylakoid membranes leads to overall water splitting in a bio-photo-electro-chemical (BPEC) cell via a simple process. Thylakoids extracted from spinach are introduced into a BPEC cell containing buffer solution with ferricyanide. Upon solar-simulated illumination, water oxidation takes place and electrons are shuttled by the ferri/ferrocyanide redox couple from the thylakoids to a transparent electrode serving as the anode, yielding a photocurrent density of $0.5 \mathrm{mAcm}^{-2}$. Hydrogen evolution occurs at the cathode at a bias as low as $0.8 \mathrm{~V}$. A tandem cell comprising the BPEC cell and a Si photovoltaic module achieves overall water splitting with solar to hydrogen efficiency of $0.3 \%$. These results demonstrate the promise of combining natural photosynthetic membranes and man-made photovoltaic cells in order to convert solar power into hydrogen fuel.

\footnotetext{
${ }^{1}$ The Nancy \& Stephen Grand Technion Energy Program (GTEP), Technion-Israel Institute of Technology, Technion City, Haifa 32000, Israel. ${ }^{2}$ Department of Materials Science and Engineering, Technion-Israel Institute of Technology, Technion City, Haifa 32000, Israel. ${ }^{3}$ Schulich Faculty of Chemistry, Technion-Israel Institute of Technology, Technion City, Haifa 32000, Israel. ${ }^{4}$ Faculty of Biology, Technion-Israel Institute of Technology, Technion City, Haifa 32000, Israel. * These authors contributed equally to this work. Correspondence and requests for materials should be addressed to A.R. (email: avner@mt.technion.ac.il).
} 
T he need for cost effective and sustainable solutions for storing intermittent solar power has spurred a growing interest in artificial photosynthesis and solar fuels ${ }^{1-3}$. The most elementary solar fuel production process relies on the water-hydrogen cycle, $\mathrm{H}_{2} \mathrm{O} \rightleftharpoons \mathrm{H}_{2}+\frac{1}{2} \mathrm{O}_{2}$. The forward reaction is work-consuming (endergonic). Under standard conditions it requires a minimum input of $237 \mathrm{~kJ}$ of entropy-free energy (that is, work) to dissociate one mole of water, which is the standard Gibbs free energy of the reaction $\left(\Delta G^{0}\right)$. The reaction products, $\mathrm{H}_{2}$ and $\mathrm{O}_{2}$ gases, serve as fuel that can be stored in separate vessels and converted back into power by the reverse reaction. Thus, $\mathrm{H}_{2}$ can be produced sustainably by water electrolysis using renewable power sources such as solar power. Replacement of the entire worldwide primary energy consumption (12,910 million tons oil equivalent in 2014; ref. 4) by solar $\mathrm{H}_{2}$ produced at an average solar to hydrogen $(\mathrm{STH})$ conversion efficiency of $1 \%$, equivalent to the solar to biomass conversion efficiency of high efficiency crop plants ${ }^{5}$, would demand a net area of 9.7 million $\mathrm{km}^{-2}$ of solar collectors. This accounts for $6.5 \%$ of the total land area or $70.3 \%$ of the estimated arable land area on earth, a scenario which is not very practical ${ }^{6}$.

This limitation motivates research on other routes to store solar energy as chemical bonds in fuel products, for example through biotechnological or artificial photosynthesis processes. Bio-processes for renewable $\mathrm{H}_{2}$ production have been explored using fermentative and photosynthetic organisms as cellular factories for $\mathrm{H}_{2}$ production. Traditionally, these bio-processes are categorized into: (i) dark fermentation, (ii) photo-fermentation and (iii) biophotolysis ${ }^{7}$. Through the fermentative processes, protons serve as electron acceptors from catabolized organic compounds, while $\mathrm{H}_{2}$ production by biophotolytic processes make use of organisms capable of oxygenic photosynthesis, for example, green microalgae or cyanobacteria. For the latter, sunlight drives the oxidation of water molecules that serve as the electron donor, and protons generated are subsequently reduced to molecular hydrogen. A major barrier to commercialization of these technologies is the necessity to impose substantial restrictions on the bioreactor operating conditions in order to generate significant quantities of $\mathrm{H}_{2} ; \mathrm{H}_{2}$ production with green algae occurs only under near anaerobic conditions, as the primary catalytic enzyme involved ((Fe-Fe)-hydrogenase) is inhibited by oxygen. Sustained $\mathrm{H}_{2}$ production using algae can be achieved only when photosynthetic $\mathrm{O}_{2}$ evolution is severely inhibited by sulfur deprivation, to such an extent that it equals the $\mathrm{O}_{2}$ consumption in the cellular respiration. Similarly, $\mathrm{H}_{2}$ production from filamentous or unicellular cyanobacteria requires the removal of $\mathrm{O}_{2}$ from the growth medium ${ }^{8}$.

The work described here puts forward a different approach that brings together light and photosynthetic membranes in a bio-photo-electro-chemical (BPEC) cell that generates, under illumination, an electromotive force that facilitates the water splitting reaction and funnels electrons and protons for the generation of $\mathrm{H}_{2}$. The operation of the BPEC cell requires the concerted operation of three components: (i) a photochemical module that captures light and produces separated charge carriers with excess chemical potential, (ii) a catalytic module that employs the photo-excited charge carriers to carry out endergonic oxidative or reductive chemistry, and (iii) an efficient method for transferring the charge carriers between the two modules while keeping as much of their excess free energy as possible ${ }^{8}$. In previous studies, photosystem I (PSI) complexes isolated from thermophilic cyanobacteria and tethered to either Pt nanoparticle catalyst ${ }^{9}$ or $[\mathrm{NiFe}]$-hydrogenase ${ }^{10}$ were reported to produce up to $170 \mathrm{~mol} \mathrm{H}_{2}$ (mol PSI min) ${ }^{-1}$, under illumination, in the presence of sacrificial electron donor (sodium ascorbate). McCormick et al. ${ }^{11}$ reported a BPEC cell that uses a two-step process to produce $\mathrm{H}_{2}$ at a rate of $2.4 \mu \mathrm{mol} \mathrm{H}_{2}(\mathrm{mg} \mathrm{Chl} \mathrm{h})^{-1}$, using live culture of the cyanobacteria Synechocystis PCC 6803, electron mediator (ferricyanide, $\mathrm{Fe}(\mathrm{III}) \mathrm{CN}$ ), and a Pt cathode. Recently, Wang et al. ${ }^{12}$ reported the overall water splitting by self-assembled photosystem II (PSII) membranes on artificial catalysts, achieving $\mathrm{H}_{2}$ production rate of $9.1 \mu \mathrm{mol} \mathrm{H}_{2}$ $(\mathrm{mgChl} \mathrm{h})^{-1}$. In this system, the PSII membranes were irreversibly attached to particles containing ruthenium or rhodium, along with other inorganic components, in the presence of $\mathrm{Fe}(\mathrm{III}) \mathrm{CN}$. In another recent study, Mersch et al. ${ }^{13}$ presented a BPEC cell that couples PSI membranes and hydrogenase to mesoporous transparent electrodes, using 2,6dichloro-1,4-benzoquinone (DCBQ) as an electron mediator. A benchmark water splitting photocurrent of $450 \mu \mathrm{A} \mathrm{cm}^{-2}$ was obtained at an applied bias of $0.9 \mathrm{~V}$ under red light illumination $(\lambda=660 \mathrm{~nm})$ at an intensity of $10 \mathrm{~mW} \mathrm{~cm}^{-2}$, corresponding to a light-to-hydrogen conversion efficiency of $(1.5 \pm 0.1) \%$.

Unlike conventional (first generation) biofuel production technologies from crop plants, a process that has been reported to work at an average efficiency of up to $1 \%$ and creates a serious competition for food ${ }^{5}$, BPEC cells make use of leaves, and are not limited to a particular plant species. Leaves of most crops do not have high commercial value, being mostly used as feedstock. We envision the utilization of crop leaves for green and benign $\mathrm{H}_{2}$ production, as an intermediate step after the crop plant has given its fruits, and before being fed to animals. Thus, in this work we present a BPEC cell that produces $\mathrm{H}_{2}$ gas using crude thylakoid membranes (henceforth called thylakoids) extracted from spinach, which is used here as a plant model. The thylakoids are extracted with minimal preparatory effort and then applied simply by letting them settle onto the surface of a transparent electrode, thereby enabling easy replacement of photodamaged thylakoids by fresh ones. Under illumination, a photocurrent is produced, mediated by $\mathrm{Fe}(\mathrm{III}) \mathrm{CN}$, and channelled to evolve $\mathrm{H}_{2}$ at the cathode at applied bias far below the reversible voltage of water electrolysis (1.23 V in standard conditions). Furthermore, we present a stand-alone mode of operation, wherein the BPEC cell is coupled in tandem to a Si photovoltaic (PV) module that provides the bias required to produce $\mathrm{H}_{2}$. Thus, $\mathrm{H}_{2}$ is produced by this hybrid tandem cell from oxidized water molecules without any external power source or sacrificial electron donors.

\section{Results}

BPEC cell. Our BPEC cell design meets the following principles: (i) Utilization of crude thylakoids without chemical immobilization or modification. This prevents the deleterious outcomes of immobilization procedures to the photosystems ${ }^{14}$, and therefore, it is expected to retain high photosynthetic activity, as well as enable easy replacement of photodamaged thylakoids; (ii) Utilization of a durable and inexpensive transparent electrode; and (iii) Use of a stable electron mediator $(\mathrm{Fe}(\mathrm{III}) \mathrm{CN}$ ) to allow efficient electron extraction from the thylakoids and fast charge transfer to the anode ${ }^{11,12}$. The use of crude thylakoids purified from spinach in a BPEC cell is schematically illustrated in Fig. 1a. The anode is a transparent electrode made of fluorinated-tinoxide (FTO) coated glass substrate onto which the thylakoids were applied simply by letting them to settle from a slurry of crude spinach thylakoids in buffer solution onto the anode. Figure $1 \mathrm{~b}$ shows scanning electron microscopy images of the thylakoids on the FTO surface. For clarity of observation, only a small amount of thylakoids was used in the preparation of the scanning electron microscopy specimen. The thylakoids were not tightly attached to the anode, and could easily be washed away by gently flushing with buffer solution. Thus, stirring of the thylakoids during operation of the BPEC cell was impossible and not deemed critical for performance. 
a

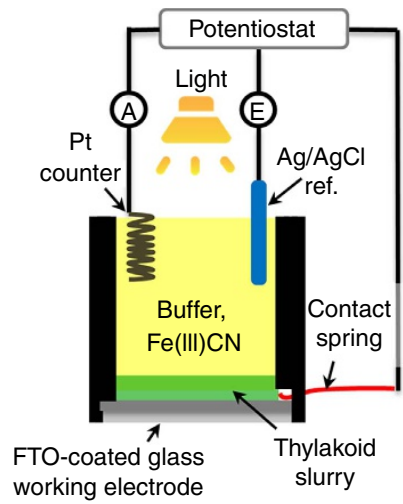

b

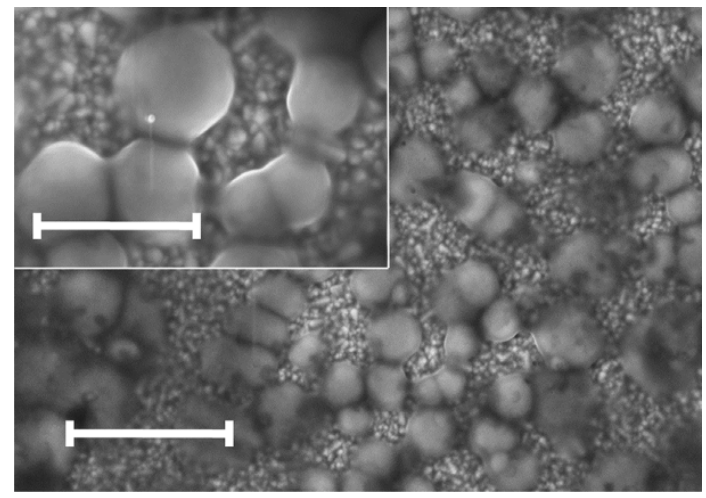

Figure 1 | The BPEC cell. (a) Schematic illustration of the BPEC cell. The cell comprises a cylindrical container with a window at the bottom, where a transparent working electrode (FTO coated glass) serving as the anode is sealed against a rubber gasket. The anode is electrically connected through a contact spring to a potentiostat, which is set in three-electrode mode (except for the two-electrode measurements described towards the end of the article), along with an $\mathrm{Ag} / \mathrm{AgCl}$ reference electrode and a Pt counter electrode (that is, cathode). The potentiostat measures the current between the working and counter electrodes using an ammeter $\{A\}$, and the potential $\{E\}$ of the working electrode with respect to that of the reference electrode. A minimal amount of thylakoids settles from a slurry of crude spinach thylakoids in buffer solution containing glycerol onto the exposed area $\left(1.08 \mathrm{~cm}^{2}\right)$ of the FTO anode. The cell cavity is filled with $20 \mathrm{ml}$ buffer A solution, containing the redox mediator Fe(III) $\mathrm{CN}\left(\mathrm{K}_{3} \mathrm{Fe}(\mathrm{CN})_{6}\right)$, which serves as a recyclable electron shuttle between the thylakoids and the anode. (b) Scanning electron microscopy image of the spinach membranes on the surface of a FTO coated glass electrode. Small amount of membranes were placed on the electrode and the scanning electron microscopy images were taken in vacuum. Scale bar, $2 \mu \mathrm{m}$. Inset: scanning electron microscopy image at higher magnification. Scale bar, $1 \mu \mathrm{m}$.
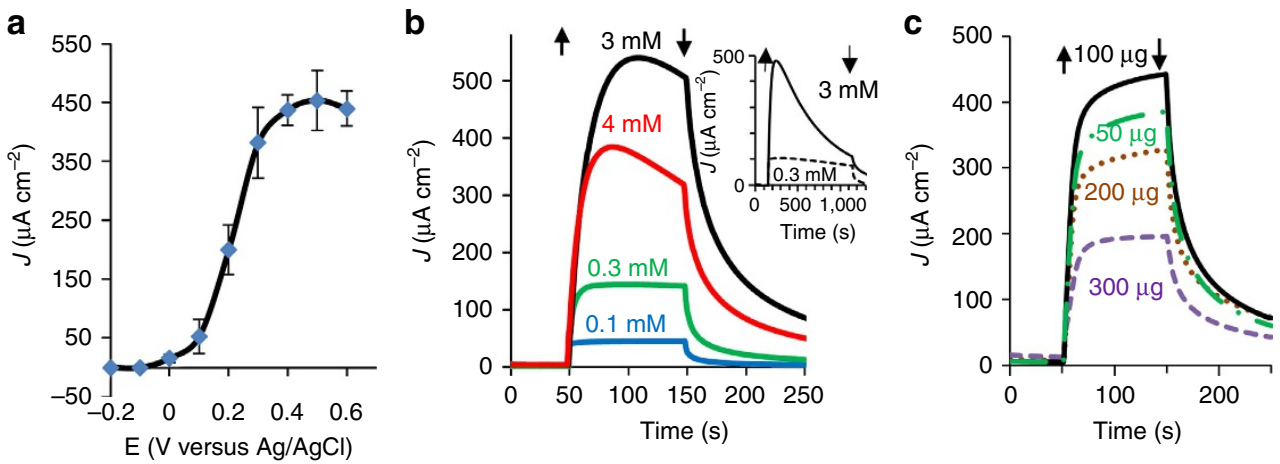

Figure 2 | Photocurrent measurements in three-electrode mode. (a) Photocurrent density as a function of the electrode potential, measured in buffer A solution with a thylakoid content of $0.1 \mathrm{mg} \mathrm{Chl}$ and $\mathrm{Fe}(\mathrm{III}) \mathrm{CN}$ concentration of $3 \mathrm{mM}$. The error bars represent the s.d. over four independent measurements. (b) The photocurrent, measured at an electrode potential of $0.5 \mathrm{~V}_{\mathrm{Ag} / \mathrm{AgCl}}$, as a function of time during exposure to solar-simulated light between $t=50$ and $150 \mathrm{~s}$. Four measurements in buffer A solution with a thylakoid content of $0.1 \mathrm{mg} \mathrm{Chl}$ and Fe(III)CN concentrations of $0.1,0.3,3$ and $4 \mathrm{mM}$ are presented (blue, green, black and red curves, respectively). The inset shows the long-term photocurrent stability in high ( $3 \mathrm{mM}$, full line) and low $(0.3 \mathrm{mM}$, dashed line) Fe(III)CN concentrations. The arrows indicate light turn on (up) and off (down) points. (c) The photocurrent dependence on the amount of thylakoids (expressed in $\mathrm{mg} \mathrm{Chl)} \mathrm{in} \mathrm{solution.} \mathrm{Maximal} \mathrm{photocurrent} \mathrm{was} \mathrm{obtained} \mathrm{with} 100 \mu \mathrm{g}$ Chl (black line), whereas 50,200 or $300 \mu \mathrm{g}$ Chl (green broken, brown dotted or purple dashed lines, respectively) yielded lower currents. The measurements were carried out in buffer A solution with $3 \mathrm{mM} \mathrm{Fe}(\mathrm{III}) \mathrm{CN}$.

Significant photocurrent from spinach thylakoids. Under solarsimulated illumination, water oxidation is catalysed by the PSII complex embedded in the thylakoids, releasing $\mathrm{O}_{2}$ and protons. A mediated photocurrent is obtained in the system by use of the redox couple ferri/ferrocyanide ( $\mathrm{Fe}(\mathrm{III}) / \mathrm{Fe}(\mathrm{II}) \mathrm{CN})$ that extracts electrons from the photosynthetic membranes and transfers them to the anode. The photocurrent dependence on the applied potential is presented in Fig. 2a, showing saturation of $450 \pm 50 \mu \mathrm{Acm}^{-2}$ at an anode potential of $0.5 \mathrm{~V}_{\mathrm{Ag} / \mathrm{AgCl}}$. The measurements were carried out in buffer A solution with a thylakoid content of $0.1 \mathrm{mgChl}$ and a $\mathrm{Fe}(\mathrm{III}) \mathrm{CN}$ concentration of $3 \mathrm{mM}$. The dependencies of the photocurrent, measured at $0.5 \mathrm{~V}_{\mathrm{Ag} / \mathrm{AgCl}}$, on the $\mathrm{Fe}(\mathrm{III}) \mathrm{CN}$ concentration (at a thylakoid content of $0.1 \mathrm{mgChl}$ ) and thylakoid content (at a Fe(III)CN concentration of $3 \mathrm{mM}$ ) are presented in Fig. 2b,c, respectively. A maximal photocurrent density of $c a .0 .5 \mathrm{~mA} \mathrm{~cm}^{-2}$ was obtained using $3 \mathrm{mM} \mathrm{Fe}(\mathrm{III}) \mathrm{CN}$ and thylakoid content of $0.1 \mathrm{mg} \mathrm{Chl}$.

Previous reports have shown photo-induced electron transfer from PSII and PSI ${ }^{15}$ and the production of photocurrent from spinach thylakoids utilizing $\mathrm{Fe}(\mathrm{III}) \mathrm{CN}$ as electron shuttle, as well as using other thylakoid sources, different types of electron mediators, and a variety of electrode compositions. For example, Calkins et al. ${ }^{16}$ immobilized spinach thylakoids onto multi-walled carbon nanotubes, and achieved a maximal photocurrent of $68 \mu \mathrm{A}$ using $\mathrm{Fe}(\mathrm{III}) \mathrm{CN}$ as the electron mediator. We have previously reported a photocurrent density of $100 \mu \mathrm{Acm}^{-2}$ using a graphite electrode and DCBQ as an electron shuttle ${ }^{17}$. Hasan et al. ${ }^{18}$ reported a maximal photocurrent density of $130 \mu \mathrm{Acm}^{-2}$ using a gold electrode and para-benzoquinone electron transfer mediator. Thylakoids have also been used 
without an electron mediator ${ }^{19,20}$, though the reported photocurrents were lower compared with reports with an electron mediator. Larom et al. ${ }^{21}$ reported the production of an unmediated photocurrent density of $16 \mu \mathrm{Acm}^{-2}$ using crude Synechocystis membranes and $\mathrm{N}$-acetyl cysteine-modified gold electrode. A photocurrent density of $42 \mu \mathrm{A} \mathrm{cm}^{-2}$ was reported by Hamidi et al. ${ }^{22}$, using immobilized spinach thylakoids in osmium polymer network on a graphite electrode. Other studies reported high photocurrents using purified PSI or PSII. A solid state electrochemical device ${ }^{23}$ that combines purified photosynthetic complexes with a transparent electrode coated with a sandwich of metallic nanolayers reached a photocurrent density of $120 \mu \mathrm{Acm}^{-2}$. This study is mentioned in a recent review article on photosynthetic protein based PV devices ${ }^{24}$ as setting the record for these devices. Isolated PSII membranes were also employed by Mersch et al. ${ }^{13}$, reporting photocurrent densities as high as $930 \mu \mathrm{Acm}^{-2}$ at an applied potential of $0.5 \mathrm{~V}$ versus the normal hydrogen electrode (NHE). However, the photocurrent in this study was measured under red light illumination $\left(\lambda=679 \mathrm{~nm}, 10 \mathrm{~mW} \mathrm{~cm}^{-2}\right)$, which makes it difficult to compare with our results that were measured under solar-simulated white light illumination. Hence, the photocurrent density reported here sets a new record for solar-simulated measurements of BPEC cells utilizing any photosynthetic membranes, crude or purified ones. Furthermore, the preparation of isolated reaction centers is expensive, time and energy consuming and requires the use of polluting detergents, and the photocurrent quickly decays due to irreversible degradation of the reaction centers ${ }^{13,16,18,19,22}$. Our approach alleviates these drawbacks by using crude thylakoids that can be readily replaced by fresh ones, as demonstrated below.

To estimate the efficiency of the charge transfer from the thylakoids to the electric circuit we calculated the ratio between the total photo-induced charge, $Q_{\text {photo }}$, measured by integrating the photocurrent $\left(I_{\text {photo }}\right)$ over time, $Q_{\text {photo }}=\int I_{\text {photo }} d t$, and the amount of $\mathrm{O}_{2}$ that evolved by the photosynthetic membranes, $\Delta \mathrm{O}_{2}$ (measured in $\left(\mathrm{mol} \mathrm{O}_{2}\right)$ ). Thus, the charge transfer efficiency $\left(\eta_{\mathrm{ct}}\right)$ was calculated according to equation (1):

$$
\eta_{\mathrm{ct}}=\frac{4 \cdot \Delta \mathrm{O}_{2} \cdot F}{Q_{\text {photo }}} \cdot 100 \%
$$

where $F$ is the Faraday constant. In order to calculate $\Delta \mathrm{O}_{2}$ we measured the increase in oxygen concentration after $10 \mathrm{~min}$ of illumination using a Clark electrode (see Supplementary Fig. 1a). The charge transfer efficiency was $(94 \pm 11) \%$ (Supplementary
Fig. 1b). This value indicates that under these conditions almost all of the electrons derived from water photo-oxidation were successfully transferred to the electric circuit.

Photoactivity lifetime. $\mathrm{Fe}(\mathrm{III}) \mathrm{CN}$ reduction by isolated chloroplasts or sub-chloroplast components is one of the early facilitators of the Hill reaction and has been known since the 1950 's. Studies have suggested that Fe(III)CN is reduced by several separate donor sites located in PSII, plastoquinone (PQ) and the cytochrome $b_{6} f$ or $\mathrm{PSI}^{25}$. Earlier studies have made clear that the reduction is accompanied by an adverse effect which rapidly inhibits photosynthesis. A dose-dependent perturbation of $20-50 \%$ in the oxygen evolution rate was reported for spinach chloroplasts that were dark-incubated in $0.5-20 \mathrm{mM} \mathrm{Fe}(\mathrm{III}) \mathrm{CN}$ for $20 \mathrm{~min}^{26}$. Furthermore, Kirilovsky et al. ${ }^{27}$ investigated the complex light-dependent PSII inactivation caused by Fe(III)CN incubation by electron paramagnetic resonance (EPR) spectroscopy and fluorescence induction techniques. Their report suggests that the inhibition is the result of a dual effect; the first one is the over-oxidation of the donor site in PSII, which uncouples the oxygen evolving center, and the second one is the formation of abnormal states of reduced $Q_{\mathrm{A}}$ at the acceptor site.

Here we study a system that exploits $\mathrm{Fe}(\mathrm{III}) \mathrm{CN}$ as an electron mediator from photo-activated thylakoids. As expected, photocurrent degradation was observed, especially at high photocurrent densities (see inset of Fig. 2b). Thus, we sought to elucidate the nature of the photocurrent degradation. Towards this end, spinach thylakoids were placed in the BPEC cell and incubated in light or dark in the presence or absence of ferricyanide (Fe(III)CN) or ferrocyanide (Fe(II)CN) as a control, and then used, subsequently, for photocurrent and photosynthetic electron flow measurements. Figure 3 a presents the residual photocurrent that was measured following $10 \mathrm{~min}$ of different incubation treatments. The residual activities are expressed as the ratio between the photocurrent and PSII activities obtained after the incubation to those obtained in a control experiment using identical thylakoids without $10 \mathrm{~min}$ of incubation. Incubations in light or dark without $\mathrm{Fe}(\mathrm{III}) \mathrm{CN}$ were found to reduce the photocurrent to about 60 and $80 \%$ of the initial photocurrent, respectively, indicating that light enhances the degradation of the photocurrent. In addition, there is significantly more reduction of the photocurrent when the thylakoids are incubated with $\mathrm{Fe}$ (III)CN in the light, resulting with only $20 \%$ of the initial photocurrent (Fig. 3a). To rule out the possibility that the reduced form of the electrolyte, that is $\mathrm{Fe}(\mathrm{II}) \mathrm{CN}$ obtained from the
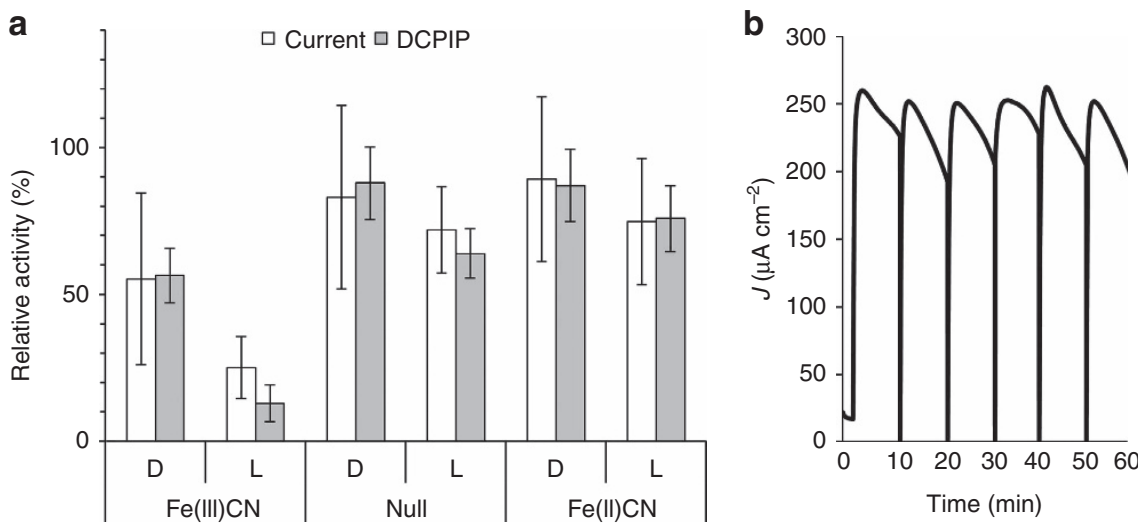

Figure 3 | Light-induced reduction of Fe(III)CN damages the photosynthetic activity of the thylakoids. (a) The residual photocurrent and DCPIP reduction obtained from thylakoids after incubation for $10 \mathrm{~min}$ in the dark (D) or in light (L), without (null) or with $3 \mathrm{mM} \mathrm{Fe(III)CN} \mathrm{or} \mathrm{Fe(II)CN,} \mathrm{normalized}$ to a control experiment using identical thylakoids without incubation. The error bars represent the s.d. over five measurements. (b) Batch mode of operation wherein damaged thylakoids were replaced with fresh ones every $10 \mathrm{~min}$. All the other cell components were reused. 
reduction of $\mathrm{Fe}(\mathrm{III}) \mathrm{CN}$ in the light, degraded the photocurrent, thylakoids were also incubated with $\mathrm{Fe}(\mathrm{II}) \mathrm{CN}$ under the same conditions. The degradations that were observed following incubation with $\mathrm{Fe}(\mathrm{II}) \mathrm{CN}$ in the dark or light were comparable to that of thylakoids that were incubated without $\mathrm{Fe}(\mathrm{III}) \mathrm{CN}$ (Fig. 3a). This observation is consistent with reports that $\mathrm{Fe}(\mathrm{II}) \mathrm{CN}$ ions are harmless to chloroplast or sub-chloroplast components in the $\mathrm{mM}$ concentration range ${ }^{28}$.

Since the BPEC cell makes use of only a small amount of thylakoids in the presence of excess $\mathrm{Fe}(\mathrm{III}) \mathrm{CN}$ in solution, we hypothesized that the light-dependent photocurrent decay may have occurred as a result of the PSII membranes reaching their turnover number limit, after which PSII was damaged. To examine this hypothesis and to determine the fraction of photosynthetically active electron flow in the thylakoids in each of the incubation conditions, thylakoids were collected from the BPEC cell after $10 \mathrm{~min}$ of incubation, the $\mathrm{Fe}(\mathrm{III}) \mathrm{CN}$ was washed out by centrifugation, the thylakoids were re-suspended in fresh medium (as explained in the experimental procedures), and analysed for 2,6-dichlorophenolindophenol (DCPIP) reduction. The results are presented in Fig. 3a. There is correlation between the photocurrent degradation and the DCPIP reduction degradation. A residual photosynthetic electron flow activity of $10 \%$ was observed for thylakoids that were incubated with $\mathrm{Fe}(\mathrm{III}) \mathrm{CN}$ in light, compared with $60 \%$ of this activity that was retained in thylakoids that were incubated in light without $\mathrm{Fe}(\mathrm{III}) \mathrm{CN}$. This observation suggests that the combination of light and $\mathrm{Fe}(\mathrm{III}) \mathrm{CN}$ is most harmful to the thylakoids, enhancing the photoinhibition of the photosynthetic electron flow. Thus, we conclude that the photocurrent degradation was caused in part by the photoinhibition deactivation of photosynthetic electron flow which is enhanced in the present of $\mathrm{Fe}$ (III)CN. Additional contribution of the $\mathrm{Fe}(\mathrm{III}) \mathrm{CN}$ to the degradation of the photocurrent and the photosynthetic electron flow occurred in the dark (Fig. 3a). In order to overcome the degradation problem, the thylakoids were replaced with fresh ones every $10 \mathrm{~min}$ in a time course of $1 \mathrm{~h}$, while continuously monitoring the photocurrent. As shown in Fig. 3b, the photocurrent was kept at a high level throughout the entire hour, except for the intermittent exchange points every $10 \mathrm{~min}$. This demonstrates that the electrodes and mediator solution may be reused for a long time.

\section{The photocurrent is derived from photosynthetic electron transfer.} To examine whether the source of electrons was indeed photosynthetic water photolysis and not spurious side reactions, the photosynthesis inhibitor 3-(3,4-dichlorophenyl)-1,1-dimethylurea (DCMU) was introduced to the system as a probe. DCMU is known to effectively block the electron transfer from $Q_{\mathrm{A}}$ to $Q_{\mathrm{B}}$ in PSII $^{29,30}$, as illustrated in Fig. 4a (see also Supplementary Fig. 2). Hence, it inhibits continuous water photo-oxidation. Figure $4 \mathrm{~b}$ shows the relative photocurrent with and without DCMU as a function of DCMU concentration. It is evident that an inhibitory effect governs the process, with a complete photocurrent arrest at $500 \mu \mathrm{M}$ DCMU. The inhibition by DCMU implies that the source of electrons reducing the $\mathrm{Fe}(\mathrm{III}) \mathrm{CN}$ mediator must be at or downstream the $Q_{\mathrm{B}}$ site, which is the site blocked by the herbicide ${ }^{30}$ (see Fig. 4a). Unlike the quinone acceptors DCBQ and DCPIP which accept electrons mostly from the quinone-binding sites of PSII $\left(Q_{\mathrm{B}}\right)$ and Cytochrome $b_{f} f$ (cyt $\left.b_{f} f\right), \mathrm{Fe}(\mathrm{III}) \mathrm{CN}$ can accept electrons from several electron donor sites under different conditions, including $Q_{\mathrm{A}}{ }^{30}$, the $\mathrm{PQ}$ pool ${ }^{31}$, cyt $b_{6} f$ and $\mathrm{PSI}^{32}$. To further investigate the electron source of the reduction of $\mathrm{Fe}(\mathrm{III}) \mathrm{CN}$ in our system, we performed a series of experiments with the photosynthesis inhibitor 2,5-dibromo-6-isopropyl-3methyl-1,4-benzoquinone (DBMIB). At low concentrations
DBMIB blocks the electron transfer from the PQ pool to cyt $b_{6} f$ (refs 30,33). This inhibition was observed in our system, as shown by the persistence of electron transfer from $\mathrm{H}_{2} \mathrm{O}$ to DCBQ but not from $\mathrm{H}_{2} \mathrm{O}$ to methyl viologen dichloride (MV) in the presence of $5 \mu \mathrm{M}$ DBMIB, see Fig. 4c. DBMIB did not inhibit the photocurrent, as well as the $\mathrm{H}_{2} \mathrm{O}$ to $\mathrm{Fe}(\mathrm{III}) \mathrm{CN}$ electron transfer rate, indicating that $\mathrm{Fe}(\mathrm{III}) \mathrm{CN}$ extracts electrons before cyt $b_{6} f$. Together with the observation that DCMU suppressed the photocurrent (Fig. 4b), namely, it blocked the $\mathrm{Fe}(\mathrm{III}) \mathrm{CN}$ reduction, the observation that DBMIB did not inhibit these photoactivities (Fig. 4c) implied that the first site to reduce $\mathrm{Fe}(\mathrm{III}) \mathrm{CN}$ along the electron transfer chain must be the PQ pool and/or the reduction of cyt $b_{6} f$ site (see Fig. 4a). Interestingly, the photocurrent increased in the presence of $5 \mu \mathrm{M}$ DBMIB (Fig. 4c), indicating that electrons were extracted from $P Q$ at a faster rate than from all the sites without the inhibitor, as reported elsewhere ${ }^{30,32}$.

Additional support to this conclusion was obtained by comparing the photoactivities of PSII particles prepared by the Berthold, Babcock and Yoccum (BBY) method (ref. 34) with those of crude thylakoids, see Fig. 4d. The photocurrent and $\mathrm{H}_{2} \mathrm{O}$ to $\mathrm{Fe}(\mathrm{III}) \mathrm{CN}$ electron transfer rate of the PSII particles were much lower than the respective values obtained with intact thylakoids (see Supplementary Fig. 3), despite the fact that PSII particles gave rise to higher specific activity when measured at a lower chlorophyll concentration than what was used here (see note in the legend of Fig. 4d). The PSII particles are enriched in PSII but contain less PQ and negligible amounts of cyt $b_{6} f$ and $\mathrm{PSI}^{34}$, compared with the thylakoids ${ }^{35}$. An additional explanation to the reduced photocurrent produced by the PSII particles as compared with that produced by intact thylakoids takes into account the reduced amount of PQ pool, which is about 3-fold lower in the PSII particles than in crude thylakoids ${ }^{35}$. The solution reduction potential is controlled by the concentrations of $\mathrm{Fe}(\mathrm{III}) \mathrm{CN}$ and $\mathrm{Fe}(\mathrm{II}) \mathrm{CN}$, and is in equilibrium with the redox poise of the PQ pool, which is smaller and its turnover rate is slower in the PSII particles. Therefore, if PQ is reducing the exogenous $\mathrm{Fe}(\mathrm{III}) \mathrm{CN}$, its reduced amount and turnover rate in the PSII particles results in a smaller photocurrent, as shown by the measurement of the $\mathrm{H}_{2} \mathrm{O}$ to $\mathrm{Fe}(\mathrm{III}) \mathrm{CN}$ electron transfer rate in Fig. 4d. Evidences for the beneficial contribution of PSI and cyt $b_{6} f$ complex to the photocurrent were reported by Calkins et al. ${ }^{16}$ and by Rasmussen and Minteer $^{36}$ in similar systems. In the latter work, it was found that the dominant electron donor was PSI, followed by PSII and the cyt $b_{6} f$ complex $^{36}$. In order to analyse whether or not $\mathrm{Fe}(\mathrm{III}) \mathrm{CN}$ is reduced by PSI in the present system, MV, which is a competitor to ferredoxin for accepting electrons from the $\mathrm{Fa} / \mathrm{Fb}$ site of $\mathrm{PSI}^{16}$, was added to the system resulting with the reduction of the photocurrent by about 30\% (see Supplementary Fig. 4). Altogether, the results in Fig. 4 show that the photocurrent was inhibited by DCMU but not by DBMIB, and that the PSII particles produced lower photocurrent than intact thylakoids, as well as the partial inhibition by MV, indicating that the mediator $\mathrm{Fe}(\mathrm{III}) \mathrm{CN}$ extracts photosynthetic electrons mostly from $\mathrm{PQ}$, and possibly also from cyt $b_{6} f$ and PSI (to a lesser extent).

Further evidence to the involvement of photosynthesis in the photoactivity was obtained by analysing the spectral response of the photocurrent. We hypothesized that the photocurrent would be proportional to the light intensity absorbed by chlorophyll, the dominant pigment in plant thylakoids. Thus, we anticipated the maximal photocurrent to be obtained at wavelengths of 460 and $660 \mathrm{~nm}$, where the light absorption of chlorophyll is maximal (see Supplementary Fig. 5). In order to examine this hypothesis, we measured the external quantum efficiency (EQE) spectrum using thylakoids in the amount equivalent to $100 \mu \mathrm{g}$ Chl placed in the cell. The results are shown in Fig. 5, displaying two peaks at 

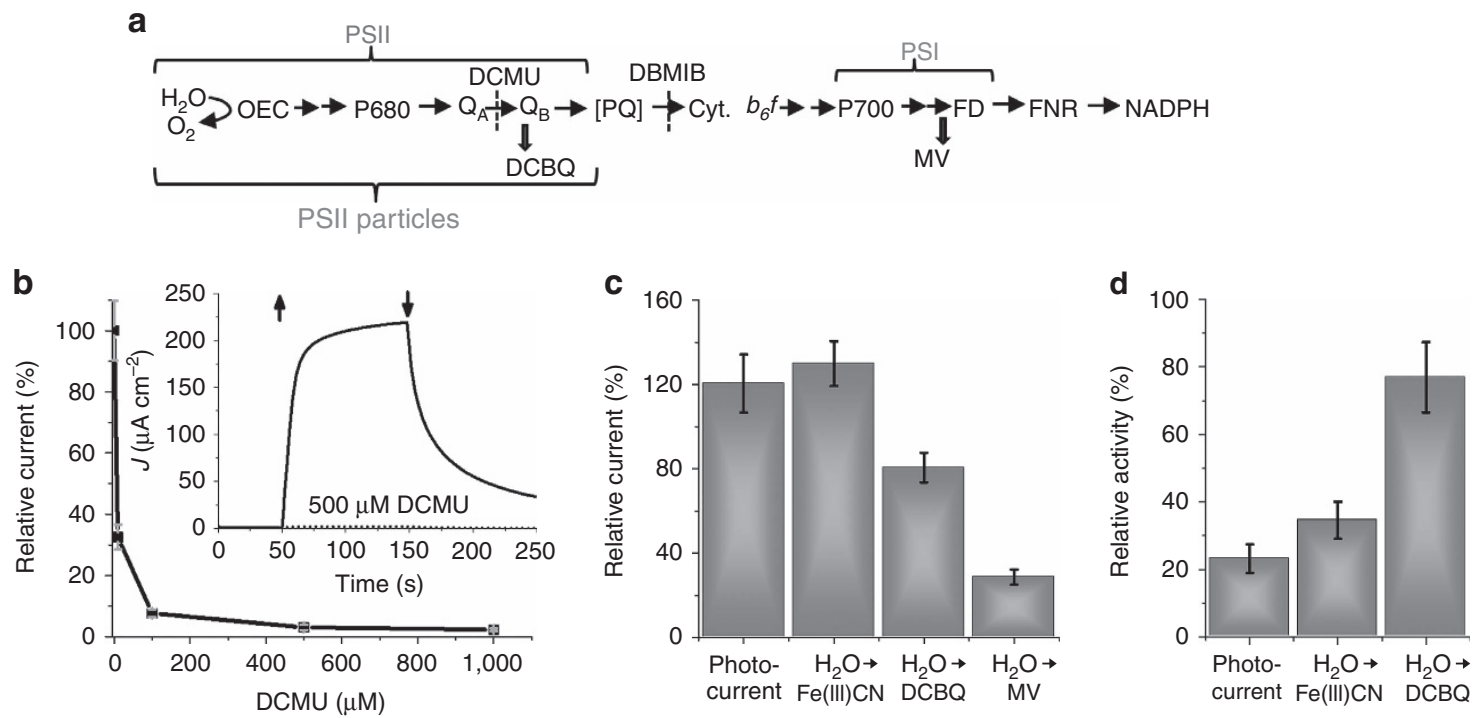

Figure 4 | The photosynthetic electrons are extracted by Fe(III)CN following the $\mathbf{Q}_{\mathbf{B}}$ site. (a) A scheme of the photosynthetic electron flow (horizontal arrows) with exogenous electron acceptors (vertical arrows) and electron flow inhibitors (vertical dashed lines). Double arrows mark additional electron acceptors. (b) Relative photocurrent of thylakoids, measured at a potential of $0.5 \mathrm{~V}_{\mathrm{Ag} / \mathrm{AgCl}}$ with $3 \mathrm{mM} \mathrm{Fe}$ (III)CN, as a function of DCMU concentration, averaged over at least three experiments. The relative photocurrent is the photocurrent measured with DCMU divided by the photocurrent measured without it, as shown in the inset. (c) Relative photoactivity measured with DBMIB ( $5 \mu \mathrm{M})$ with respect to measurements without DBMIB. The columns show (left to right) the relative photocurrent and oxygen evolution rates measured with Fe(III)CN or DCBQ as acceptors and the oxygen consumption rate using MV as electron acceptor. The results error bars represent the s.d. over at least three measurements. (d) Relative photocurrent and photoactivity of $\mathrm{Fe}(\mathrm{III}) \mathrm{CN}$ and $\mathrm{DCBQ}$ reduction rates in PSII particles, relative to the respective measurements with intact thylakoids (both at the equivalent amount of $0.1 \mathrm{mg}$ (hl). It is noted that when measured at the equivalent amount of lower chlorophyll concentration such as $0.01 \mathrm{mg} C h l$, the $\mathrm{H}_{2} \mathrm{O}$ to DCBQ activity of the PSII particles was higher than that of intact thylakoids. The error bars represent the s.d. over at least three measurements.

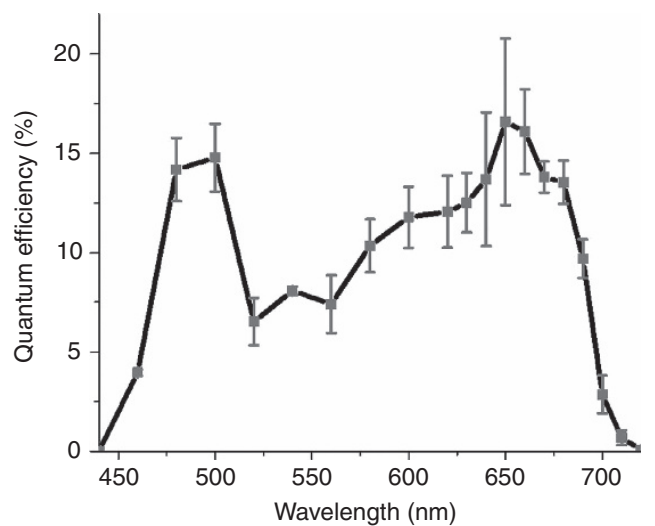

Figure 5 | Quantum efficiency. The quantum efficiency, that is, the photo induced electrons per photons, measured at various wavelengths from 440 to $720 \mathrm{~nm}$ with a spectral bandwidth of $10 \mathrm{~nm}$. Measurements were taken with different samples of thylakoids $(0.1 \mathrm{mg} \mathrm{Chl})$ for each wavelength. The photocurrent was measured at a potential of $0.5 \mathrm{~V}_{\mathrm{Ag} / \mathrm{AgCl} \text {. The values }}$ error bars represent the s.d. over three measurements.

the same wavelengths as the peaks in the absorption spectrum of the spinach thylakoids (Supplementary Fig. 5). A maximum EQE of $(17 \pm 4) \%$ was obtained at $650-660 \mathrm{~nm}$. A similar value was reported by Mersch et al. ${ }^{13}$, using mesoporous transparent electrodes with a much larger amount of PSII than we used here.

Low bias hydrogen generation. Photoelectrochemical water splitting has been proposed as a promising strategy for capturing and storing solar energy in the form of chemical energy, for example hydrogen fuel ${ }^{37}$. Towards this end, we sought the conditions in which the photocurrent could give rise to the reduction of protons in order to form $\mathrm{H}_{2}$ at the cathode. Thus, the anode and cathode were connected to the potentiostat in twoelectrode mode of operation, and the photocurrent was measured as a function of the voltage between the two electrodes. In addition, the potential of the cathode was monitored simultaneously by measuring the voltage drop between the cathode and a reference electrode, as illustrated in Supplementary Fig. 6a. This enabled simultaneous measurement of the photocurrent as function of the applied voltage between the anode and cathode, as well as the potentials of the two electrodes. The results are shown in Fig. 6a. For hydrogen evolution, the cathode must be at a potential lower than $0 \mathrm{~V}$ with respect to the reversible hydrogen electrode (RHE) scale. This condition is reached at a minimum voltage of $0.8 \mathrm{~V}$ (see Fig. 6a), where the onset of the water photoelectrolysis reaction takes place. In a control experiment using the same setup without the thylakoids, a voltage of $2 \mathrm{~V}$ was required to initiate the water electrolysis reaction (see cyclic voltammogram in Supplementary Fig. 6b). Furthermore, in the experiment without the thylakoids the potential of the cathode was higher than $0 \mathrm{~V}_{\mathrm{RHE}}$ in the entire bias range of $0-1.5 \mathrm{~V}$ (see Supplementary Fig. 6c), indicating that no hydrogen evolution occurred in this range. Thus, the introduction of thylakoids to the BPEC cell reduced the minimum bias for the onset of the water splitting reaction from 2 to $0.8 \mathrm{~V}$. The onset at $0.8 \mathrm{~V}$ is equal to the difference between the redox potential of the $\mathrm{Fe}(\mathrm{III}) / \mathrm{Fe}(\mathrm{II}) \mathrm{CN}$ redox couple and the hydrogen evolution reaction (HER) potential at a Pt electrode. This indicates that the light reactions split water and reduce $\mathrm{Fe}(\mathrm{III}) \mathrm{CN}$, thus the $\mathrm{Fe}(\mathrm{III}) / \mathrm{Fe}(\mathrm{II}) \mathrm{CN}$ redox couple sets the anode potential, whereas the cathode potential reaches the HER potential at the onset of hydrogen evolution, giving rise to a bias of $0.8 \mathrm{~V}$ at the onset of water splitting with hydrogen evolution at the cathode. It is noteworthy that some photocurrent was measured below the onset of the water splitting reaction (see Fig. 6a). This photocurrent arose from other electrochemical side 

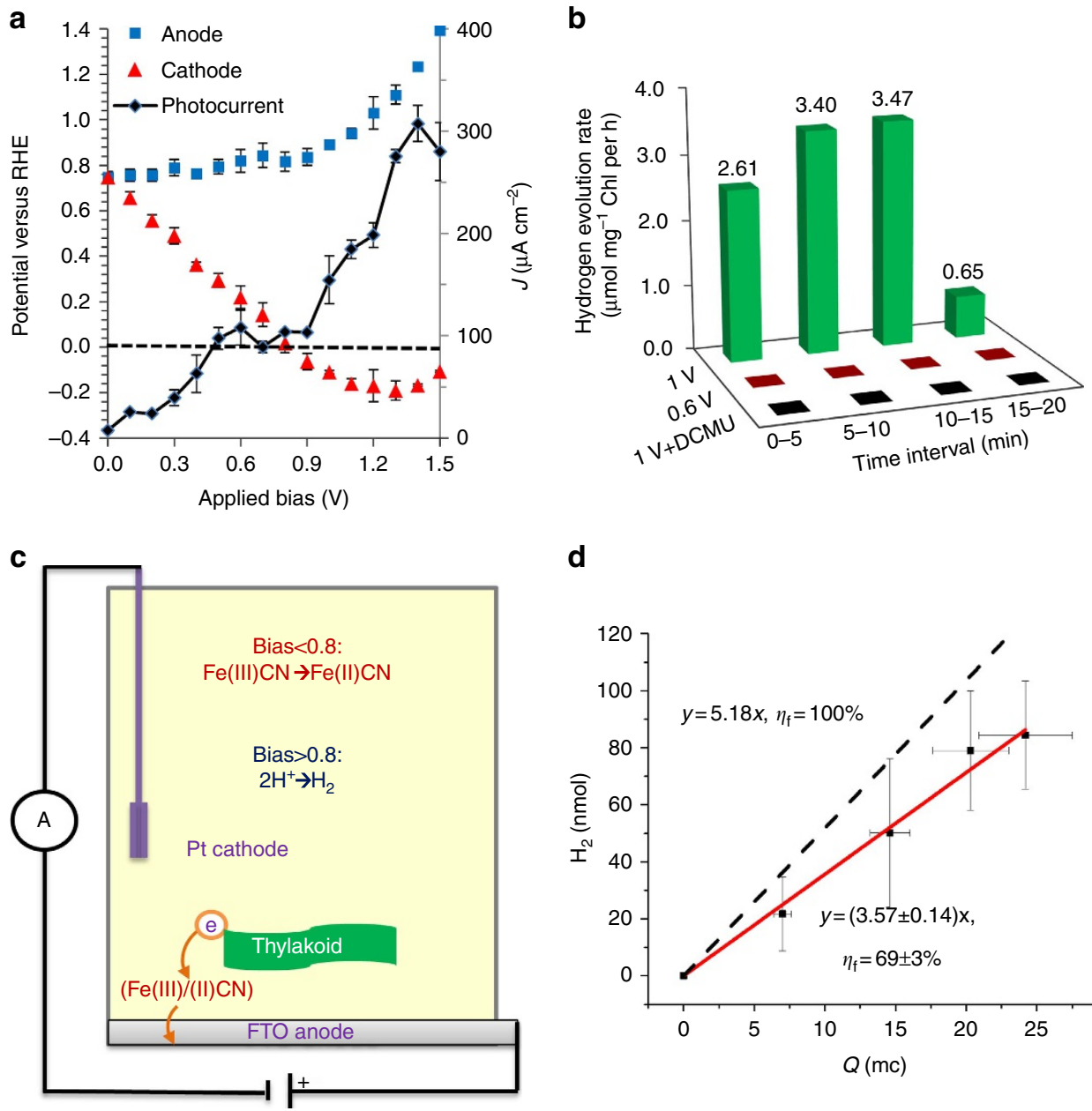

d

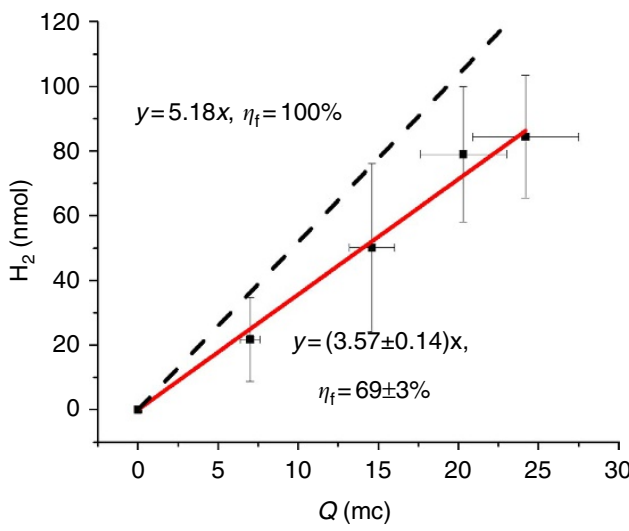

Figure 6 | Two-electrode mode measurements. (a) Photocurrent (black curve) and anode (blue squares) and cathode (red triangles) potentials as a function of the applied bias between the anode and cathode. The dashed line represents a potential of $O V_{R H E}$. The photocurrent was measured under exposure to solar-simulated light in a cell containing buffer A solution with a thylakoid content of $0.1 \mathrm{mg} \mathrm{Chl}$ and Fe(III)CN concentration of $3 \mathrm{mM}$. The error bars represent the s.d. over three independent measurements (b) Hydrogen evolution rate at the cathode under a bias of $0.6 \mathrm{~V}$ (red bars), $1.0 \mathrm{~V}$ (green bars) and $1.0 \mathrm{~V}$ with the addition of $0.5 \mathrm{mM} \mathrm{DCMU}$. (c) Schematic illustration of the proposed electron transfer pathway in the BPEC cell. When the applied bias is lower than $0.8 \mathrm{~V}$ the cathode reaction is dominated by cyclic electron transfer of the Fe(III)CN/Fe(II)CN couple, whereas above $0.8 \mathrm{~V}$ proton reduction to hydrogen prevails. (d) $\mathrm{H}_{2}$ production as a function of the charge that was transferred between the anode and cathode. Dashed black line corresponds to a Faradic efficiency of $100 \%$. The error bars represent the s.d. over four independent measurements.

reactions, most likely the redox cycle of the $\mathrm{Fe}(\mathrm{III}) / \mathrm{Fe}(\mathrm{II}) \mathrm{CN}$ couple. The photocurrent associated with these processes saturated at $\sim 100 \mu \mathrm{A} \mathrm{cm}^{-2}$ at a bias of $0.5 \mathrm{~V}$ (see Fig. 6a). Between 0.5 and $0.8 \mathrm{~V}$ the photocurrent was constant, starting to increase again above $0.8 \mathrm{~V}$ and reaching $\sim 300 \mu \mathrm{A} \mathrm{cm} \mathrm{cm}^{-2}$ at $1.4 \mathrm{~V}$. Thus, the net water photoelectrolysis current density reached approximately $200(=300-100) \mu \mathrm{Acm}^{-2}$ at $1.4 \mathrm{~V}$, indicating that the Faradaic efficiency for the water splitting reaction was ca. $67 \%$.

In order to confirm this indirect estimation, the gases that evolved at the cathode headspace were collected and analysed by gas chromatography (GC). The $\mathrm{H}_{2}$ evolution rate at several time intervals during GC measurements at a bias of 0.6 or $1.0 \mathrm{~V}$ are presented in Fig. 6 b. The $\mathrm{H}_{2}$ evolution rate between 5 and $15 \mathrm{~min}$ was found to be $3.4-3.5 \mu \mathrm{mol}(\mathrm{mg} \mathrm{Chl} \mathrm{h})^{-1}$, being among the highest reported values for BPEC devices that operate without sacrificial electron donors. In control experiments, under a bias of $1 \mathrm{~V}$ and in the presence of $0.5 \mathrm{mM}$ DCMU, or in the presence of $\mathrm{Fe}(\mathrm{III}) \mathrm{CN}$ but without thylakoids, $\mathrm{H}_{2}$ production could not be detected at all (Fig. $6 \mathrm{~b}$ and Supplementary Fig. 6c), demonstrating that electron flow down-stream of PSII is crucial for $\mathrm{H}_{2}$ production. These observations are summarized in a schematic illustration of the proposed electron transfer pathways in the cell as depicted in Fig. 6c. Finally, the Faradaic efficiency $\left(\eta_{\mathrm{F}}\right)$ is calculated from the ratio between the slops of the $\mathrm{H}_{2}$ versus $\mathrm{Q}$ (Fig. 6d), indicating that the total charge that transfers between the working and counter electrodes produces hydrogen at a $(69 \pm 3) \%$ efficiency. This value is consistent with the dual electron pathway suggested in Fig. $6 c$, wherein $2 / 3$ of the photocurrent supports proton reduction at the cathode, whereas the remaining $1 / 3$ feeds the $\mathrm{Fe}(\mathrm{III}) / \mathrm{Fe}(\mathrm{II}) \mathrm{CN}$ electron transfer cycle.

Hybrid BPEC-PV tandem cell for solar water splitting. The photo-activated spinach thylakoids in the BPEC cell significantly reduced the voltage needed to produce $\mathrm{H}_{2}$ from water. Nonetheless, an external bias of at least $0.8 \mathrm{~V}$ was still needed to energize the process. Aiming to construct a stand-alone solarpowered device that does not require an external power source to operate, the BPEC cell was coupled in tandem with a Si PV cell (see photograph in Fig. 7a) that provides the bias needed for the 

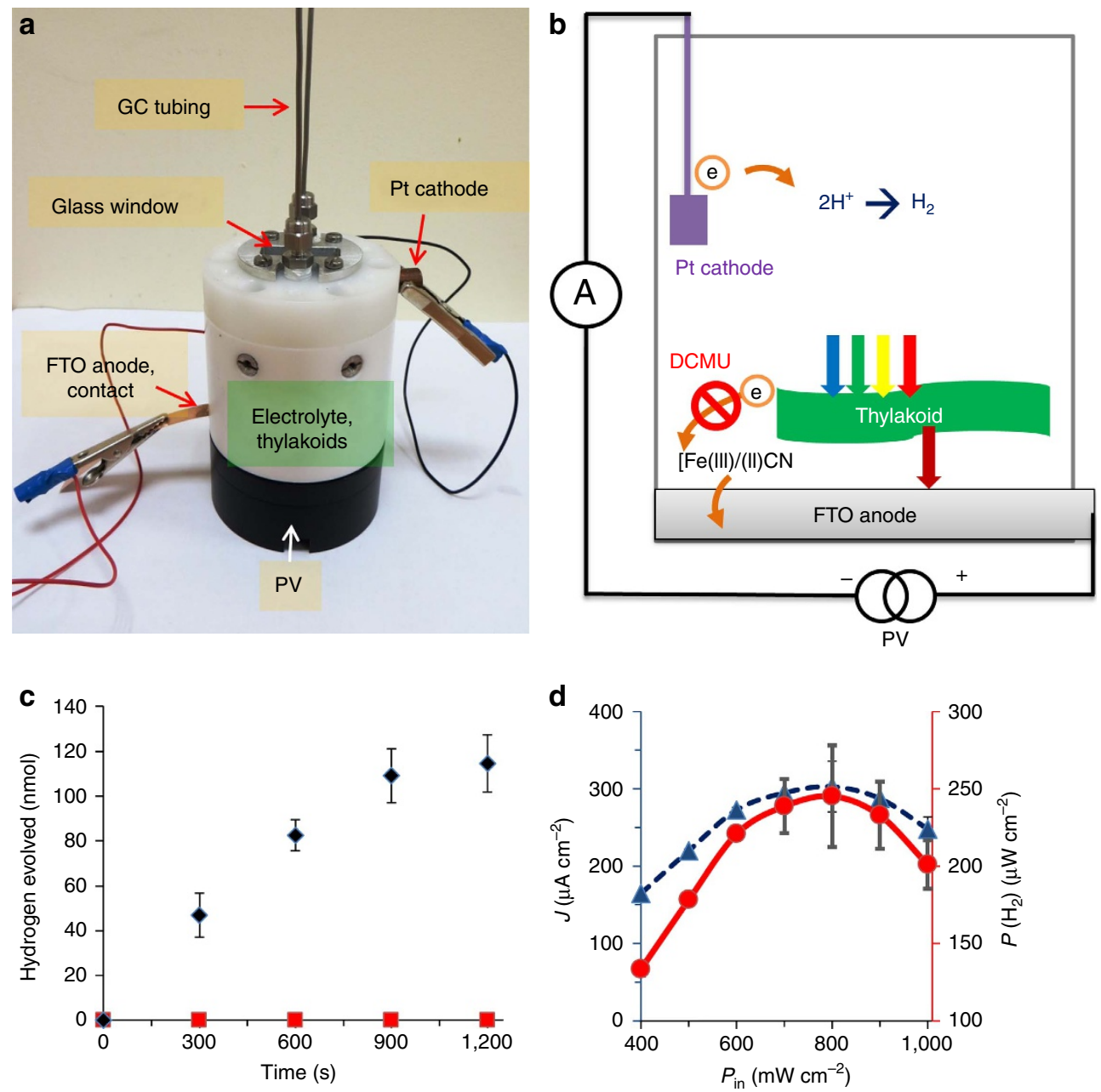

Figure 7 | PV-BPEC tandem cell. (a) Photograph of the tandem cell. (b) Illustration of the electron transfer pathway in the tandem cell. (c) Hydrogen evolution at the cathode in the presence (red squares) or absence (black diamonds) of the herbicide DCMU. (d) The influence of the incident light intensity $\left(P_{\text {in }}\right)$ on the photocurrent (blue curve) and power accumulated in hydrogen bonds (red curve) produced by the tandem cell. The error bars in $\mathbf{c}, \mathbf{d}$ represent the s.d. in four independent measurements.

water splitting reaction, as illustrated in Fig. 7b. This design mimics the Z-scheme in photosynthesis, wherein PSI reenergizes the electrons from PSII. In the BPEC-PV tandem cell, water oxidation is energized by the short wavelength radiation of the sunlight spectrum, harvested by the thylakoids. In addition, the long wavelength radiation transmitted through the thylakoids is captured by the PV cell, activating it to produce the power needed to support the overall water splitting reaction. In other words, the PV cell replaces the potentiostat that was used in the experiments described in the previous sections. Such a tandem design has two important merits in regards to solar water splitting. First, coupling the cells in series to increase the photovoltage produced by the device enables overall water splitting, which neither one of the cells could drive alone. Second, unlike a single absorber system, where only photons possessing sufficient energy to drive the water photolysis reaction are used to stimulate the PV cell, in the tandem cell less energy is needed from each photon. This allows a larger wavelength threshold (that is, more efficient light harvesting) and smaller thermalization loss, thereby increasing the power conversion efficiency of the system ${ }^{38,39}$. To evaluate the performance of the hybrid BPEC-PV tandem cell, the gases produced in the BPEC cell were analysed by GC (Fig. 7c). $\mathrm{H}_{2}$ gas formation was observed only in the presence of all the components, namely the electrolyte solution, thylakoids and PV cell. Maximal $\mathrm{H}_{2}$ production rate was obtained within the first
15 min of operation, after which the rate decreased and finally plateaued after $20 \mathrm{~min}$. These observations are consistent with the results obtained for the BPEC cell alone (see inset of Fig. $2 \mathrm{~b}$ and Fig. 6b). Expectedly, the addition of the herbicide DCMU totally inhibited the $\mathrm{H}_{2}$ evolution (see Fig. 7c).

Last, the effect of light intensity on the photocurrent produced by the BPEC-PV tandem cell was examined. The power output of the cell was calculated according to equation (2):

$$
P_{\text {out }}=\eta_{\mathrm{F}} \times J_{\text {photo }} \times \Delta G^{0}
$$

where $\eta_{\mathrm{F}}$ is the faradaic efficiency for the water splitting reaction (69\%, see Fig. $6 \mathrm{~d}), J_{\text {photo }}$ is the photocurrent density and $\Delta G^{0}$ is the chemical potential of the water splitting reaction $(1.23 \mathrm{~V}$ per electron in standard conditions). The maximal photocurrent density and power output of the cell were obtained under illumination of $80 \mathrm{~mW} \mathrm{~cm}^{-2}$ (see Fig. 7d), yielding $J_{\text {photo }}=300 \pm 25 \mu \mathrm{A} \mathrm{cm}^{-2}$ and $P_{\text {out }}=250 \pm 20 \mu \mathrm{W} \mathrm{cm}^{-2}$. This corresponds to STH conversion efficiency, $\eta_{\mathrm{STH}}=P_{\mathrm{out}} / P_{\text {in }}$ where $P_{\text {in }}$ is the power density of the incident light, of $(0.31 \pm 0.03) \%$. It is noteworthy that the hybrid BPEC-PV tandem cell reached maximum performance at $80 \mathrm{~mW} \mathrm{~cm}^{-2}$, less than full 1 Sun conditions $\left(100 \mathrm{~mW} \mathrm{~cm}^{-2}\right)$. The decrease in photocurrent at light intensities above $80 \mathrm{~mW} \mathrm{~cm}^{-2}$ (see Fig. 7d) is most likely due to photo-saturation of the thylakoids at this light intensity threshold. 


\section{Discussion}

We presented here a BPEC cell comprising of plant thylakoids in electrolyte solution that produced photocurrents as high as $0.5 \mathrm{~mA} \mathrm{~cm}^{-2}$ derived from photosynthetically catalysed water oxidation. Powering the cell with additional bias greater than $0.8 \mathrm{~V}$ leads to overall water splitting with hydrogen and oxygen production at a Faradic efficiency of $(69 \pm 3) \%$. A stand-alone tandem cell comprising the BPEC cell and a Si PV cell was demonstrated, reaching STH conversion efficiency of $0.3 \%$. This innovative hybrid system brings together natural photosynthetic membranes and man-made PV technology in order to solve one of the greatest challenges in renewable energy development, that is solar energy conversion and storage in hydrogen fuel. Further development of this promising approach should aim to enhance the STH conversion efficiency, for example, by replacing flat transparent electrodes with porous ones.

\section{Methods}

Plant material and thylakoid membrane isolation. Spinach (Spinacia oleracea) grown in fields of the lower Galilee between the coordinates $33^{\circ} 4^{\prime} 18.4^{\prime \prime} \mathrm{N}$ to $32^{\circ} 51^{\prime} 50.5^{\prime \prime} \mathrm{N}$ and $35^{\circ} 16^{\prime} 32.8^{\prime \prime} \mathrm{E}$ to $35^{\circ} 6^{\prime} 24.2^{\prime \prime} \mathrm{E}$ was obtained from the local growers. The results presented in this work were obtained using three independent biological preparations. The thylakoid membranes preparation was carried out by grinding the young leaves in buffer A $(50 \mathrm{mM}$ 2-(N-morpholino)ethanesulfonic acid $(\mathrm{MES}) / \mathrm{NaOH} \mathrm{pH}=6.0,15 \mathrm{mM} \mathrm{NaCl}, 5 \mathrm{mM} \mathrm{MgCl}, 2 \mathrm{mM} \mathrm{CaCl}_{2}$ ), and precipitating the thylakoids according to the procedure described by Andreasson et $a l .{ }^{40}$ PSI I enriched membrane fragments (BBY membranes) were extracted using the detergent Triton X-100 (Sigma), as described by Berthold et al. ${ }^{34}$ The chlorophyll content in thylakoid preparations was determined according to the procedure described by Arnon ${ }^{41}$.

Photoelectrochemical measurements. The photocurrent produced by the thylakoids was measured in the BPEC cell illustrated in Fig. 1a. The measurements were carried out at ambient temperature $\left(25 \pm 2{ }^{\circ} \mathrm{C}\right) .0 .1 \mathrm{mg} \mathrm{Chl} a$ of the thylakoids in buffer A solution containing $10 \% \mathrm{v} / \mathrm{v}$ glycerol were set on a FTO-coated glass electrode placed at the bottom of the BPEC cell. The BPEC cell was illuminated from the top by a solar simulator (Oriel Sol3A class AAA solar simulator, Newport, USA) calibrated to provide solar simulated (AM1.5G) light intensity of 1 Sun $\left(100 \mathrm{~mW} \mathrm{~cm}^{-2}\right)$ at the BPEC cell. The operating conditions were chosen based on a series of cyclic voltammetry measurements, and carried out using a potentiostat (Zennium, ZAHNER-elektrik, Germany) connected either in a three-electrode mode with an $\mathrm{Ag} / \mathrm{AgCl}$ (in $3 \mathrm{M} \mathrm{NaCl}$ solution) reference electrode (RE-1B, $\mathrm{CH}$ Instruments, USA) and a platinum wire counter electrode, or in a two-electrode mode to a platinum wire counter electrode. Fe(III)CN mediated photocurrents were measured chrono-amperometrically at different potentials in buffer A solutions with different amounts of potassium ferricyanide $\left(\mathrm{K}_{3} \mathrm{Fe}(\mathrm{CN})_{6}\right.$, Sigma Aldrich) as described in the text, under cyclic exposure to light-dark pulses. In order to examine the electron source, the herbicides DCMU (Fluka) and DBMIB (Aldrich) were used. The photocurrent density was calculated as the quotient of the current under illumination minus the dark current, divided by the projected area of the electrode $\left(1.08 \mathrm{~cm}^{2}\right)$ that was exposed to the light and in direct contact with the solution containing the thylakoids (Fig. 1a). The standalone solar water splitting tests were carried out by coupling the BPEC cell in tandem with a Si PV module (IXOLAR XOB17-04x3 SolarBit, IXYS, Korea). The photocurrent between the working (TEC15) and counter (Pt wire) electrodes and the voltage drop between the working and reference $(\mathrm{Ag} / \mathrm{AgCl})$ electrodes were monitored by digital multimeters (34401A DMM, Agilent Technologies, USA) and recorded digitally using LabVIEW (National Instruments, USA).

Quantum efficiency measurements. The EQE of the BPEC cell with $0.1 \mathrm{mg}$ Chl and $3 \mathrm{mM} \mathrm{Fe}$ (III)CN was measured at a potential of $0.5 \mathrm{~V}_{\mathrm{Ag} / \mathrm{AgCl}}$. The thylakoids were illuminated by monochromatic light obtained by coupling a white light source to a monochromator (Cornerstone CS260, Newport, USA) with a spectral band width of $10 \mathrm{~nm}$. Fresh thylakoid membranes were used in each measurement, and the maximum photocurrent was recorded. This was repeated at different wavelengths, three times for each wavelength. The photon flux was obtained from the light intensity measured by a power meter (918D High Performance Photodiode Sensor, Newport, USA), and the EQE was calculated according to equation (3):

$$
\mathrm{EQE}=\frac{\text { Maximum photocurrent } / q}{\text { Photon flux }}
$$

Photoinhibition measurements. Photoinhibition experiments were done in the BPEC cell described above and with the same conditions as the photocurrent and hydrogen evolution measurements. Membranes were collected from the cell after 10 min incubation in the different conditions: dark/light, $\pm \mathrm{Fe}$ (III)CN or $\pm \mathrm{Fe}(\mathrm{II}) \mathrm{CN}$. The thylakoids were re-suspended in fresh buffer to wash out the $\mathrm{Fe}$ (III)CN. Ten percent of the sample was taken for the measurement of the photosynthetic electron flow by spectroscopic analysis of the rate of DCMU and light dependent reduction of DCPIP (Sigma) serving as an indicator for oxygen production ${ }^{42}$, and the remaining $90 \%$ was used for photocurrent measurements. The photocurrent was measured using three electrode mode voltammetry (Ivium-n-Stat multichannel potentiostat, Ivium Technologies, The Netherlands) in fresh medium (buffer A, $3 \mathrm{mM} \mathrm{Fe}(\mathrm{III}) \mathrm{CN}, 0.09 \mathrm{mg} \mathrm{Chl}$ ) under solar simulated illumination (model 10500 solar simulator, ABET Technologies, USA). DCPIP reduction was measured in $1 \mathrm{ml}$ cuvette containing: buffer A, 50 $\mu \mathrm{M}$ DCPIP, thylakoids $(10 \mu \mathrm{g} \mathrm{Chl})$. The sample was illuminated with a white light halogen projector (0.15SU lamp, MRC). The DCPIP concentration was measured every $20 \mathrm{~s}$ with a monochromatic illumination at $600 \mathrm{~nm}$ for $100 \mathrm{~s}$ (using CO8000 cell density meter, WPA Biowave). The DCPIP reduction and the photocurrent values were normalized to the values measured when the membranes were placed in the BPEC cell (without $\mathrm{Fe}(\mathrm{III}) \mathrm{CN}$ ) and immediately removed, centrifuged, re-suspended and divided $10 \%$ to DCPIP and $90 \%$ to photocurrent measurements.

$\mathbf{O}_{2}$ evolution and consumption. For comparison with the photocurrent measurements the $\mathrm{O}_{2}$ evolution rate was measured in a Clark electrode (Hansatech Instruments, UK) with $0.1 \mathrm{mg} \mathrm{Chl} \mathrm{membranes} \mathrm{and} 3 \mathrm{mM} \mathrm{Fe}(\mathrm{III}) \mathrm{CN}$ as an electron acceptor. The sample was illuminated with a solar simulator (model 10500 solar simulator, ABET Technologies, USA) at 1 Sun for $10 \mathrm{~min}$. For other measurements the $\mathrm{O}_{2}$ evolution rate was calculated for $0.1 \mathrm{mg}$ Chl with $3 \mathrm{mM}$ DCBQ, Fe(III)CN or 1,1' $1^{\prime}$-dimethyl-4,4' -bipyridinium (MV, Aldrich) as electron acceptors with or without DCMU and DBMIB, under white light illumination (MRC). Reduced MV reduces the $\mathrm{O}_{2}$ and therefore it measures the consumption rate of $\mathrm{O}_{2}$.

$\mathbf{H}_{\mathbf{2}}$ evolution quantification. The $\mathrm{H}_{2}$ evolved at the cathode was collected into a designated glass tube, which was perforated at the bottom to allow buffer transfer, and sealed at the top with a rubber septum. Samples were collected from the headspace of the cell using a gas-tight syringe, and analysed by GC (7890A GC, Agilent Technologies, USA). The Faradaic efficiency for $\mathrm{H}_{2}$ generation was calculated from the ratio between the amount of $\mathrm{H}_{2}$ generated in the cell $\left(n_{\mathrm{H}_{2}}\right.$, number of $\mathrm{H}_{2}$ moles generated during time interval $t$ ) and the amount of charge $\left(Q=\int_{0}^{t} I d t^{\prime}\right)$ that was transferred between the working and counter electrodes during the same time interval $(t)$ according to equation (4):

$$
\eta_{F}=\frac{2 n_{\mathrm{H}_{2}} \cdot F}{Q} \cdot 100 \%
$$

Data availability. Data supporting the findings of this study are available within the article and its supplementary information file and from the corresponding author upon request.

\section{References}

1. Barber, J \& Tran, P. D. From natural to artificial photosynthesis. J. R. Soc. Interface 10, 20120984 (2013).

2. Cook, T. R. et al. Solar energy supply and storage for the legacy and nonlegacy worlds. Chem. Rev. 110, 6474-6502 (2010).

3. Lewis, N. S. \& Nocera, D. G. Powering the planet: chemical challenges in solar energy utilization. Proc. Natl Acad. Sci. USA 103, 15729-15735 (2006).

4. British-Petroleum. Statistical review of world energy. Available at http:// www.bp.com/en/global/corporate/energy-economics/statistical-review-ofworld-energy.html (2015).

5. Zhu, X.- G., Long, S. P. \& Ort, D. R. Improving photosynthetic efficiency for greater yield. Annu. Rev. Plant Biol. 61, 235-261 (2010).

6. Michel, H. The nonsense of biofuels. Angew. Chem. Int. Ed. 51, 2516-2518 (2012)

7. Azwar, M., Hussain, M. \& Abdul-Wahab, A. Development of biohydrogen production by photobiological, fermentation and electrochemical processes: a review. Renew. Sust. Energ. Rev. 31, 158-173 (2014).

8. Lubner, C. E. et al. Solar hydrogen-producing bionanodevice outperforms natural photosynthesis. Proc. Natl Acad. Sci. USA 108, 20988-20991 (2011).

9. Utschig, L. M., Silver, S. C., Mulfort, K. L. \& Tiede, D. M. Nature-driven photochemistry for catalytic solar hydrogen production: a photosystem I-transition metal catalyst hybrid. J. Am. Chem. Soc. 133, 16334-16337 (2011).

10. Krassen, H. et al. Photosynthetic hydrogen production by a hybrid complex of photosystem I and [NiFe]-hydrogenase. ACS Nano. 3, 4055-4061 (2009).

11. McCormick, A. J. et al. Hydrogen production through oxygenic photosynthesis using the cyanobacterium Synechocystis sp. PCC 6803 in a biophotoelectrolysis cell (BPE) system. Energy Environ. Sci. 6, 2682-2690 (2013).

12. Wang, W., Chen, J., Li, C. \& Tian, W. Achieving solar overall water splitting with hybrid photosystems of photosystem II and artificial photocatalysts. Nat Commun. 5, 4647 (2014) 
13. Mersch, D. et al. Wiring of photosystem II to hydrogenase for photoelectrochemical water splitting. J. Am. Chem. Soc. 137, 8541-8549 (2015).

14. Purcell, M., Carpentier, R., Bélanger, D. \& Fortier, G. Immobilized plant thylakoid membranes as a biosensor for herbicides. Biotechnol. Tech. 4, 363-368 (1990).

15. Agostiano, A., Goetze, D. C. \& Carpentier, R. Photoelectrochemistry of thylakoid and sub-thylakoid membrane preparations: cyclic voltammetry and action spectra. Electrochim. Acta 38, 757-762 (1993).

16. Calkins, J. O., Umasankar, Y., O’Neill, H. \& Ramasamy, R. P. High photo-electrochemical activity of thylakoid-carbon nanotube composites for photosynthetic energy conversion. Energy Environ. Sci. 6, 1891-1900 (2013).

17. Pinhassi, R. I. et al. Photosynthetic membranes of synechocystis or plants convert sunlight to photocurrent through different pathways due to different architectures. PLOS ONE 10, e0122616 (2015).

18. Hasan, K. et al. Photoelectrochemical communication between thylakoid membranes and gold electrodes through different quinone derivatives. ChemElectroChem. 1, 131-139 (2014).

19. ArianitaáDewi, H. Investigation of electron transfer from isolated spinach thylakoids to indium tin oxide. RSC Advances 4, 48815-48820 (2014).

20. Lee, J. \& Kim, S. Mediator-free solar energy conversion by the artificially installed thylakoid membrane on the functionalized electrode. Electrochem. Commun. 49, 55-59 (2014)

21. Larom, S. et al. The photosystem II D1-K238E mutation enhances electrical current production using cyanobacterial thylakoid membranes in a biophotoelectrochemical cell. Photosynth. Res. 126, 161-169 (2015).

22. Hamidi, H. et al. photocurrent generation from thylakoid membranes on osmium-redox-polymer-modified electrodes. ChemSusChem. 8, 990-993 (2015).

23. Das, R. et al. Integration of photosynthetic protein molecular complexes in solid-state electronic devices. Nano Lett. 4, 1079-1083 (2004).

24. Ravi, S. K. \& Tan, S. C. Progress and perspectives in exploiting photosynthetic biomolecules for solar energy harnessing. Energy Environ. Sci. 8, 2551-2573 (2015).

25. Barr, R., Troxel, K. S. \& Crane, F. L. EGTA, a calcium chelator, inhibits electron transport in photosystem II of spinach chloroplasts at two different sites. Biochem. Biophys. Res. Commun. 92, 206-212 (1980).

26. Brewer, J. M. \& Jagendorf, A. T. Damage to spinach chloroplasts induced by dark preincubation with ferricyanide. Plant Physiol. 40, 303 (1965).

27. Kirilovsky, D., Rutherford, A. W. \& Etienne, A. L. Influence of DCMU and ferricyanide on photodamage in photosystem II. Biochemistry 33, 3087-3095 (1994).

28. Macdowall, F. \& Lumry, R. Mechanism of the photochemical activity of isolated chloroplasts: VII. Inactivation of a photoact by dilute ferricyanide ion. Plant Physiol. 50, 305-307 (1972).

29. Cady, C. W., Crabtree, R. H. \& Brudvig, G. W. Functional models for the oxygen-evolving complex of photosystem II. Coord. Chem. Rev. 252, 444-455 (2008).

30. Trebst, A. Inhibitors in the functional dissection of the photosynthetic electron transport system. Photosynth. Res. 92, 217-224 (2007).

31. Melis, A. Functional properties of Photosystem II $\beta$ in spinach chloroplasts. Biochim. Biophys. Acta Bioenergetics 808, 334-342 (1985).

32. Ouitrakul, R. \& Izawa, S. Electron transport and photophosphorylation in chloroplasts as a function of the electron acceptor. II. Acceptor-specific inhibition by KCN. Biochim. Biophys. Acta Bioenergetics 305, 105-118 (1973).

33. Roberts, A. G., Bowman, M. K. \& Kramer, D. M. The inhibitor DBMIB provides insight into the functional architecture of the Qo site in the cytochrome $b_{6} f$ complex. Biochemistry 43, 7707-7716 (2004).

34. Berthold, D. A., Babcock, G. T. \& Yocum, C. F. A highly resolved, oxygenevolving photosystem II preparation from spinach thylakoid membranes: EPR and electron-transport properties. FEBS Lett. 134, 231-234 (1981).

35. Kurreck, J., Seeliger, A., Reifarth, F., Karge, M. \& Renger, G. Reconstitution of the endogenous plastoquinone pool in photosystem II (PS II) membrane fragments, inside-out-vesicles, and PS II core complexes from spinach. Biochemistry 34, 15721-15731 (1995).

36. Rasmussen, M. \& Minteer, S. D. Investigating the mechanism of thylakoid direct electron transfer for photocurrent generation. Electrochim. Acta 126, 68-73 (2014).
37. Brillet, J. et al. Highly efficient water splitting by a dual-absorber tandem cell. Nat. Photonics 6, 824-828 (2012).

38. De Vos, A. Detailed balance limit of the efficiency of tandem solar cells. J. Phys D: Appl. Phys. 13, 839 (1980).

39. Bolton, J. R., Strickler, S. J. \& Connolly, J. S. Limiting and realizable efficiencies of solar photolysis of water. Nature 316, 495 (1985).

40. Andreasson, E., Svensson, P., Weibull, C. \& Albertsson, P.-Å. Separation and characterization of stroma and grana membranes-evidence for heterogeneity in antenna size of both photosystem I and photosystem II. Biochim. Biophys. Acta Bioenergetics 936, 339-350 (1988).

41. Arnon, D. I. Copper enzymes in isolated chloroplasts. Polyphenoloxidase in Beta vulgaris. Plant Physiol. 24, 1-15 (1949).

42. West, J. \& Hill, R. Carbon dioxide and the reduction of indophenol and ferricyanide by chloroplasts. Plant Physiol. 42, 819-826 (1967).

\section{Acknowledgements}

This work was funded by the I-CORE Program of the Planning and Budgeting Committee, the Israel Science Foundation (grant no. 152/11), a grant from the transformative program of the US-Israel Binational Science Foundation (BSF grant no. 2011556), and a grant from the Deutsche Forschungsgemeinschaft (DIP project LU315/17-1). The results reported in this work were obtained using central facilities at the Technion's Photovoltaic Laboratory, supported by the Nancy \& Stephen Grand Technion Energy Program (GTEP) and by the Russell Berrie Nanotechnology Institute (RBNI), and at the Technion's Hydrogen Technologies Research Laboratory (HTRL), supported by the Adelis Foundation and by the Solar Fuels I-CORE. We thank Dr Anatoly Bekkerman and the Faculty of Chemistry technical team for constructing the photocell; Dr Guy Ankonina from the PV lab for assistance in design and execution of electrochemical tests; Dr Nurit Waldman and Dr Shany Hershkovitz from the hydrogen lab for assistance in quantification the hydrogen production. R.P., D.K. and G.Sa. are supported by fellowships of the Nancy \& Stephen Grand Technion Energy Program (GTEP). R.P. and D.K. are supported by fellowships of the Israeli Ministry of Science, Technology and Space. G.Sa. is supported by a Schulich Graduate fellowship.

\section{Author contributions}

R.P., N.A., G.Sc. and A.R. conceived the idea. R.P., D.K., G.Sa., H.D., N.A., G.Sc. and A.R. designed the experiments. R.P., G.Sa. and D.K. performed the main experiments. A.L. contributed to the thylakoid preparations and electrochemical experiments. A.K. performed the scanning electron microscopy imaging. V.L. contributed to the preparation of BBY. A.R., N.A., G.Sc., R.P., H.D., G.Sa. and D.K. analysed the data. R.P., A.R., N.A., G.Sc., G.Sa. and D.K. wrote the paper. A.R., N.A. and G.Sc. supervised the entire research.

\section{Additional information}

Supplementary Information accompanies this paper at http://www.nature.com/ naturecommunications

Competing financial interests: The authors declare no competing financial interests.

Reprints and permission information is available online at http://npg.nature.com/ reprintsandpermissions/

How to cite this article: Pinhassi, R. I. et al. Hybrid bio-photo-electro-chemical cells for solar water splitting. Nat. Commun. 7:12552 doi: 10.1038/ncomms12552 (2016).

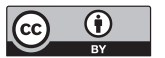

This work is licensed under a Creative Commons Attribution 4.0 International License. The images or other third party material in this article are included in the article's Creative Commons license, unless indicated otherwise in the credit line; if the material is not included under the Creative Commons license, users will need to obtain permission from the license holder to reproduce the material. To view a copy of this license, visit http://creativecommons.org/licenses/by/4.0/

(C) The Author(s) 2016 